\title{
Investigating Media Streaming in Multipath Multihop Wireless Network
}

\author{
Binod Vaidya ${ }^{1}$, SangDuck Lee ${ }^{2}$, Eung-Kon Kim ${ }^{3}$, JongAn Park ${ }^{2}$, \\ and Seung. Jo $\operatorname{Han}^{2, \star}$ \\ ${ }^{1}$ Dept. of Electronics \& Computer Eng., Tribhuvan Univ., Nepal \\ vaidyab@aol.com \\ ${ }^{2}$ Dept. of Information \& Communication Eng., Chosun Univ., Korea \\ dandylsd@hanmail.net, japark@chosun.ac.kr, sjbhan@chosun.ac.kr \\ ${ }^{3}$ Dept. of Computer Science, Sunchon National Univ., Korea \\ kek@sunchon.ac.kr
}

\begin{abstract}
Mobile Ad hoc Networks (MANETs) are very attractive for many applications. However, media streaming over MANET is quite challenging task. In this paper, we depict a framework for audio streaming over multihop wireless network. And we propose multipath routing for MANET and investigated media streaming using different scalable speech coding techniques. With the simulation results, performance of such a framework is evaluated 1
\end{abstract}

\section{Introduction}

As mobile ad hoc networks (MANETs) are self-organizing, rapidly deployable, and have no centralized control and administration, they are very attractive for many applications, such as battlefield communication, personal area networking and search-and-rescue. However, media streaming over MANET is quite challenging because of dynamic network topology, limited wireless bandwidth, and high bit error rate of wireless links.

When real-time multimedia is streamed over MANET, packet loss rate can be very high under adverse conditions, thus in turn, communication may be lost. In this paper, we propose a multipath routing protocol for multihop wireless network that can be used efficiently for media streaming. Till now, many multipath routing protocols have been proposed for wireless ad hoc networks. Some of multipath protocols based on AODV [1] are AOMDV [2], NDMR [3], and AODV-BR [4]. While streaming multimedia through multiple paths, the content can be divided into multiple minor flows and stream through available paths as in MDSR [5]. A scalable speech coding technique [6] is considered as it is designed for adaptable real-time traffics over lossy networks. Main intention of our investigation is to show the effect of different scalable speech coding techniques on MANET using proposed multipath routing protocol.

\footnotetext{
* Corresponding author.

${ }^{1}$ This study was supported (in part) by research funds from Chosun University 2006. 


\section{Multipath Routing for Media Streaming over MANET}

Proposed multipath routing protocol for MANET is basically intended for highly dynamic ad hoc networks in which communication faults frequently occur. It is basically modification of a single-path on-demand AODV routing protocol. As in AODV, three control messages are route request (RREQ), route reply (RREP) and route error (RERR). The proposed protocol has two basic phases, namely route discovery and route maintenance.

First, to find routes for a destination node $\mathrm{D}$, a source node $\mathrm{S}$ broadcasts RREQ packet. RREQ packet structure of the proposed scheme is same as that of AODV, except presence of path accumulation list of the route path. The source node appends own address to route path in RREQ. Further, when RREQ is forward by intermediate nodes, each node appends its address to it. As RREQ ID and source address form a unique identifier for RREQ, a node checks a received RREQ if it is from same source and with same RREQ ID. If a node receives first RREQ packet, it records a reverse route in its routing table. An intermediate node receiving RREQ, replies by sending a RREP if it has a route to the destination. In our proposed scheme, intermediate nodes forward duplicate RREQs that came from at most two different neighbors. This is essential to discover a number of alternate route paths.

In this scheme, the destination is responsible for selecting multiple alternate route paths. When receiving first RREQ, the destination records route paths of RREQ. Then after copying route paths of RREQ to a RREP packet, the destination node sends it to source node via its route paths. When the destination receives a duplicate RREQ, it will compare route paths of RREQ to that of the routing table. If only source node and destination node are same between them, a path is node-disjoint with primary path. If at least one of intermediate nodes in route paths in the routing table is different from all of nodes in route paths of RREQ, a route is partially disjoint path, which is defined as fail-safe [7]. Similarly, the destination sends RREP to the source along route path of RREP.

In case of route maintenance, the proposed scheme is capable of recovering broken routes immediately. When a node fails to deliver data packets to the next hop, it removes entries with broken link in its routing table and if it has another entry for the destination, data packets is delivered through the alternate route. If it has no another entry, it sends a RERR packet to the upstream node. When the source has no entry for the destination, it would initiate a new route discovery.

Scalable speech coding consists of a minimum rate bit stream that provides acceptable coded speech quality, along with one or more enhancement bit streams, which when combined with a lower rate coded bit stream, provide improved speech quality. The standards for scalable speech coding are G.727 [8], and MPEG-4 speech coding [9]. The G.727 speech coding is based upon adaptive differential pulse code modulation (ADPCM) with data rates of $16 \mathrm{kbps}$ to $40 \mathrm{kbps}$ [8]. The core bitrate is $16 \mathrm{kbps}$, and up to three $8 \mathrm{kbps}$ enhancement layers can be included. MPEG-4 Natural Speech Coding Tool Set [9] provides a generic speech coding framework having bitrate from $2 \mathrm{kbps}$ to $23.4 \mathrm{kbps}$. MPEG-4 speech 
coding scheme uses CELP (Code excited linear predictive coding) for bitrates higher than $3.85 \mathrm{kbps}$. The bitrate scalability of the core layer bitrate is possible up to three enhancement layers.

In this framework, we have evaluated G.727 scheme with 16kbps core layer and one $8 \mathrm{kbps}$ enhancement layer, for a total of $24 \mathrm{kbps}$ and MPEG-4 CELP scheme with a $6 \mathrm{kbps}$ core layer and one $2 \mathrm{kbps}$ enhancement layer, for a total of $8 \mathrm{kbps}$. Since we use scalable speech coding for media streaming, the source node begins to send core bit stream on primary path and enhancement bit stream on node-disjoint path. Since primary path and node-disjoint path are not correlated, source node uses node-disjoint path to provide load balancing. When forwarding paths break, nodes receiving core bit stream or enhancement bit stream may use different paths in the routing table to forward packets. Generally, a multihop wireless path is up or down for random periods of time, leading to bursty packet losses. A core layer packet loss is likely to be experiencing packet loss burst. So the proposed multipath protocol finds an alternate fail-safe path for each node on primary path as it has higher packet delivery rate.

\section{Performance Evaluations}

In order to evaluate performance of the proposed framework for media streaming over multipath wireless ad hoc network, we have designed experimental model and simulated using OPNET Modeler [10]. In the simulation, MANET consists of sixteen mobile nodes which are randomly placed inside a $600 \mathrm{~m}$ x $600 \mathrm{~m}$ region initially. We consider that a mobile node moves around continuously with using random waypoint mobility model with pause time of 0 s and a maximum speed of $5 \mathrm{~m} / \mathrm{s}$. The channel has a bandwidth of $1 \mathrm{Mbps}$. Transmission range is $250 \mathrm{~m}$. Among these nodes, one is randomly chosen as a streaming source and another as the destination. Five UDP traffic flows are introduced as background traffics. Each of these flows has traffic rate of four packets per second. Size of data payload is 512 bytes. Each of nodes has queue size of 10 packets.

For the experimental purpose, two scenarios have been considered, that is, a framework for audio streaming over MANET using G.727 scheme whereas another using MPEG-4 speech coding technique; in both cases, the proposed multipath routing scheme is used. Performance metrics computed during simulations are packet loss rate and end-to-end packet delay.

In order to analyze the simulation results for above framework, we compare performance of two different scalable speech coding techniques in terms of packet loss rate with respect to bit error rate (BER) and end-to-end delay (latency). Fig. 1 shows the packet loss rate for the both scenarios with respect to BER. It can be seen that the packet loss rate in G.727 coding scheme increases rapidly than in case of MPEG-4 CELP scheme. At BER of $10^{-3}$, packet loss rate for G.727 coding scheme is about 10\% whereas for MPEG-4 CELP scheme it is about $8.1 \%$. Fig. 2 illustrates the end-to-end delay for scalable speech coding techniques. It can be seen that end-to-end delay for G.727 coding scheme has higher average end-to-end delay than that of MPEG-4 CELP scheme. It can 


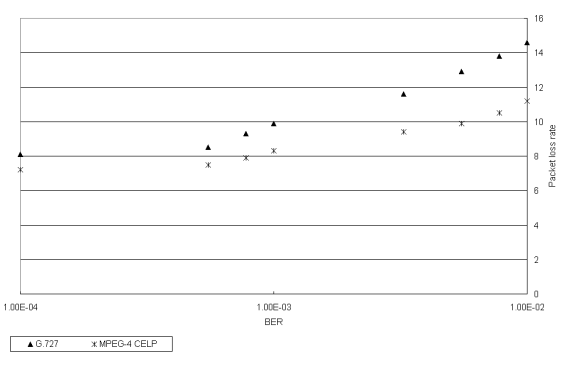

Fig. 1. Packet loss rate

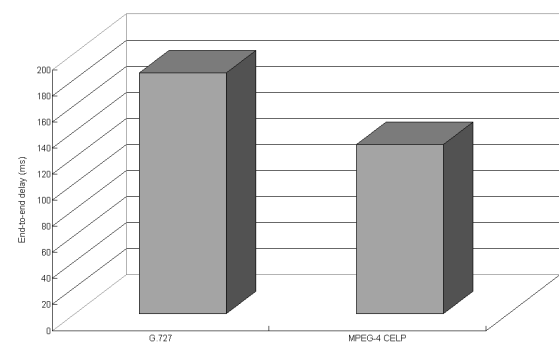

Fig. 2. End-to-end Delay

be derived that for the audio streaming over MANET, MPEG-4 CELP shows better performance than with G.727 coding scheme.

\section{Conclusions and Future Work}

In this paper we have depicted a framework for media streaming over multipath wireless ad hoc network. In order to investigate the performance of G.727 and MPEG-4 CELP speech coding schemes, we have simulated above framework using proposed multipath routing in MANET. It can be seen that the performance of MPEG-4 CELP scheme is much better that G.727 in terms of packet loss rate and end-to-end delay. In the future work, we will investigate above framework while applying selective encryption in scalable speech coding technique.

\section{References}

1. C. E Perkins, E. Royer: Ad-hoc on-demand distance vector routing, IEEE WMCSA 1999, Feb. 1999, pp 90-100

2. M.K Marina, S.R Das: Ad hoc on-demand multipath distance vector routing, Wiley Wireless Communications and Mobile Computing, Vol 6(7), 2006, pp. 969-988

3. X. Li, L. Cuthbert: Stable node-disjoint multipath routing with low overhead in mobile ad hoc networks, IEEE MASCOTS 2004, Oct 2004, pp. 184-191

4. S.J Lee, M. Gerla: AODV-BR: Backup routing in ad hoc networks, IEEE WCNC 2000, Vol 3, Sep 2000, pp. 1311-1316

5. S. Mao, et al.: Video transport over ad hoc networks: multistream coding with multipath transport, IEEE Journal on Selected Areas in Communications, Vol 21(10), Dec. 2003, pp. 1721-1737

6. H. Dong, et al.: SNR and bandwidth scalable speech coding, IEEE ISCAS 2002. Vol 2, pp. 859-862

7. L. R Reddy, S.V Raghavan: SMORT: Scalable multipath on-demand routing for mobile ad hoc networks, Elsevier Ad hoc Networks, Vol 5(2), 2007, pp. 162-188

8. ITU-T, 5-, 4-, 3-, and 2-bit/sample embedded adaptive differential pulse code modulation (ADPCM), Dec. 1990

9. ISO/IEC JTC1 SC29/WG11, ISO/IEC FDIS 14496-3, "Coding of Audio-Visual Objects Part 3: Audio", Oct. 1998

10. OPNET Modeler Simulation Software, http://www.opnet.com 